\title{
Drug Pricing and Trust: the Time Is Right
}

\author{
Julie Babyar' ${ }^{1}$ (D)
}

Published online: 22 March 2017

(C) Springer Science+Business Media New York 2017

Drug pricing and economic analyses on pharmaceuticals are most often focused on country, advocacy interest, or specialty classification. There is no one system that countries or pharmaceutical companies follow in pricing drugs. The World Health Organization has recently begun to explore fair pricing strategies for countries, as a complete and full understanding of pharmaceutical prices has yet to be explained [1] and current research detailing these systems is insufficient. Global reports and country comparisons offer some insight, but much of the available research and analyses reveal contradictory recommendations alongside challenged results. The lack of understanding in pharmaceutical pricing coupled with the lack of transparency in the industry creates maximum effort in analytics and research. The time is right to redesign this effort, create consensus on practice and policy and make decisions with the resources currently available.

The pharmaceutical and greater biomedical industries are global, and every aspect of drug pricing and access is interconnected. Pharmaceutical company sales, research and development are heavily concentrated in North America and Europe, with Asia being a third primary region of industry focus [2]. It has been noted that more than one third of the industry market share belongs to ten pharmaceutical companies, each making between $\$ 18$ billion and $\$ 70$ billion in revenue. Additionally, the industry enjoys large and increasing profit margins [3]. Within recent years, the entire pharmaceutical industry spent over $\$ 100$ billion on research and development and $\$ 3.5$ billion on treatments donated or sold at

Julie Babyar

jbabyar@gmail.com

1415 Herondo Street, Hermosa Beach, CA 90254, USA cost to underprivileged communities [4]. Pharmaceutical and drug services, research and product distribution are heavily regulated within safety aspects, contested in impact on individual wellbeing and often mistrusted by the general public. Additionally, large profits within the industry add to cynicism and concern from the public. Pricing and consequences surrounding it are of utmost importance, for both economic wellbeing as well as public interest. While proposed solutions are numerous, origins of data analytics are debated and severity of impact contested, it is the public's confidence that must be the primary priority.

Debates and contested analyses are important discussions in effort to achieve accuracy on drug pricing and access. The fact that the USA spends the most per capita on pharmaceuticals is not contested, nor are current rising prices debated. The USA spends $9.8 \%$ of its national health expenditure on pharmaceuticals [5], and $17 \%$ of personal health care services for an individual in the USA are directed toward prescription medications [6]. The impact and prioritization of drug pricing, and whether or not this is a new issue, are of debate. One recent country comparison analysis demonstrated that drug prices follow national income levels comparatively, though some price differences were apparent [7].

Drug pricing is waxes and wanes at the forefront of healthcare industry discussion. However, ongoing delivery and payment reforms as well as recent negative media attention on questionable pharmaceutical industry ethics fuel concern. Often these discussions are public, with advocacy groups representing various interests. Pharmaceutical Research and Manufacturers of America (PhRMA) cite that it costs about $\$ 2.6$ billion to develop a new medicine [8], a number that the Campaign for Sustainable Rx Pricing cites as not validated in analyses [9], and a number far larger than the $\$ 1.2$ billion average quoted by the International Federation of Pharmaceutical Manufacturers and Associations [4]. 
Drug pricing causation and effects are contested as well. An Elsevier white paper points to industry consolidation, drug and material shortages and regulation inefficiencies at the Federal Drug Administration as the primary reasons for generic drug price increases [10], while other studies point to lack of competition among pharmaceutical industries as a reason for generic price increases [6]. Specialty drug pricing is also debated, with industry associations citing drug development costs as a rationale for high drug pricing. Other expert opinions argue that high pricing of specialty drugs is an industry risk response [11], with insurance industry experts citing specialty drugs as $25 \%$ of total drug spending. These experts quote total drug spending at $\$ 87$ billion and state concerns over future large populations utilizing specialty drugs [12] without documented actuarial forecast. Orphan drug incentive policies have also been debated, with some advocates underscoring significant profit from questionable treatments [9]. Additionally, in overall drug pricing debates, patent protections are named as a reason for high prices [6] as well as a reason for the availability of new and innovative treatments through investment [8]. Finally, lack of negotiability and lack of governmental price control within the USA, compared to other countries, is cited as a primary reason for high drug prices [6] and debated in industry.

Solutions to improve drug costs are just as diverse and incongruent. Negotiating and price control abilities for the USA government are advocated by some, while other associations look to alternative strategies. Similarly, while the USA government cannot negotiate or centrally set prices, strategies such as rebate programs and newly evolving comparative effectiveness research can be strengthened to demand pricing reflect value for the public and individual patient. Regulatory improvements to the Federal Drug Administration have been encouraged by advocacy groups, including efficiency and operational reform. Strengthening competition through incentives for new players in the pharmaceutical industry has been suggested. Reforming patent protections to reflect true innovation and strengthening clinical trial and post-market surveillance is another proposed solution. Physician education to assist in appropriate prescribing has also been shown to be evidentially effective [6]. Strong development of laws and policies alongside consistent interpretation and enforcement can assist in drug pricing as well as appropriate generic and brand name balance. As research and analytics on healthcare, costs and international agenda bloom, impact data can only be interpreted accurately if laws are consistent and enforced.

Similarly, economic models that explain price differences can only be evaluated, modified, and researched accurately if the data is accurate. Price discrimination, the Ramsey pricing model, and peak load pricing model have all been reviewed as potential explanations in drug price differencing among countries, and these evaluations are only as strong as the consistency in system application that produces the data [7].
Ongoing analyses and research on drug pricing is important, and the ability to create ongoing international data collection and evaluation is possible.

As policy think tanks and global agencies drive future discussions and guidance on pharmaceuticals, pricing and understanding can be better analyzed, thoroughly understood and communicated in a comprehensive and clear manner to the public. International trade and increasing global business will call for stronger coordination and cooperation in pharmaceutical safety and regulation. As comparative effectiveness research and evidentiary standards become structured, agencies and governments will align with understanding that drug effectiveness and benefits to wellness are not based on borders. While governed peoples should continue to be free to elect variable economic models for healthcare, evidence from better research will leave less room for doubt and debate. Research and analytics on the treatments themselves as well as pharmaceutical pricing causation and effect, alongside transparency from the global pharmaceutical industry, provides the public options for evidence based decisions and secures better trust between the industry and the public.

Collective agencies, governments, and pharmaceutical companies must remain aware that, while creating a larger system of data collection and analysis, current climate requires action and decision. Equally, actions and decisions must be made with the resources at disposal, these being current data, analyses, reports, and recommendations. Because failure is not an option, and there will not be a perfect solution to an imperfect system, the decision to act requires choosing the best and most feasible response.

A full understanding of pharmaceutical pricing and implementation of best practices provides cost effective approaches, a better distribution of treatments for the global population and methodology for consensus on individual country policies that cooperate with worldwide systems. This understanding will increase responsibility and accountability on pharmaceutical industries and their stakeholder partners, including academic researchers and third party payers, in collaboration for better healthcare innovation and fairer financial burden distribution. A thorough and global consensus will also create equal knowledge distribution for all healthcare industry participants. Most importantly, this transparency and understanding on future sound research and analytics will provide a healthier trust between the industry and the public. There is nothing more critical to the healthcare industry than the public's trust, and the time is right to maximize this trust through consensus, practice, policy and appropriate resource utilization.

Compliance with Ethical Standards This manuscript has not been published elsewhere and is not under submission elsewhere and there is no conflict of interest. All manuscript preparation, literature review, writing, and analysis were completed by the author. This author reviewed and approved this manuscript for submission. All relevant ethical safeguards have been secured. 


\section{References}

1. Kieny M-P. A comprehensive and fair solution to the price of medicines. http://www.who.int/mediacentre/commentaries/fair-pricemedicines/en/. Published 2016.

2. Rugman A, Brain C. Regional strategies of multinational pharmaceutical firms. Manag Int Rev. 2004;44.

3. Anderson R. Pharmaceutical industry gets high on fat profits. http:// www.bbc.com/news/business-28212223. Published 2014. Accessed September 4, 2016.

4. Jenner A. Trends in the pharmaceutical industry and potential impact on future innovation and access to medicines. 2012.

5. Centers for Disease Control and Prevention (CDC). Fast Stats. http://www.cdc.gov/nchs/fastats/health-expenditures.htm. Published 2016. Accessed September 3, 2016.

6. Kesselheim AS, Avorn J, Sarpatwari A. The high cost of prescription drugs in the United States. JAMA. 2016;316(8):858. doi:10. 1001/jama.2016.11237.
7. Schweitzer SO, Comanor WS. Prices of pharmaceuticals in poor countries are much lower than in wealthy countries. Health Aff. 2011;30(8):1553-61. doi:10.1377/hlthaff.2009.0923.

8. Homepage. PhRMA. http://www.phrma.org/. Published 2016. Accessed September 3, 2016.

9. Proposals for Change: The Campaign for Sustainable Rx Pricing. National Coalition on Healthcare Action Fund. Published 2016. http://www.csrxp.org/proposals-for-change-transparencycompetition-and-value/. Accessed 3 Sept 2016.

10. Generic drug price increases: causes and impact (white paper). Elsevier Clinical Solutions. 2015. 1-6.

11. Stossel TP. Prescription drug pricing: scam or scapegoat? American Enterprise Institute. https://www.aei.org/publication/prescriptiondrug-pricing-scam-or-scapegoat/. Published 2016. Accessed 3 Sept 2016.

12. Kleinke JD, McGee N. Breaking the bank: three financing models for addressing the drug innovation cost crisis. Am Heal Drug Benefits. 2015;8(3). 